\title{
Protein tyrosine phosphatase receptor type $O$ expression in the tumor niche correlates with reduced tumor growth, angiogenesis, circulating tumor cells and metastasis of breast cancer
}

\author{
ZHAO LIU $^{1 *}$, JIAJIE HOU ${ }^{2 *}$, LIDONG REN ${ }^{1}$, JING HE $^{1}$, BEICHENG SUN ${ }^{2}$, \\ LU-ZHE SUN ${ }^{3}$ and SHUI WANG ${ }^{1}$
}

${ }^{1}$ Department of Breast Surgery and ${ }^{2}$ Liver Transplantation Center, The First Affiliated Hospital of

Nanjing Medical University, Nanjing, Jiangsu 210029, P.R. China; ${ }^{3}$ Department of Cellular

and Structural Biology, University of Texas Health Science Center, San Antonio, TX 78229-3900, USA

Received November 12, 2014; Accepted January 14, 2015

DOI: $10.3892 /$ or.2015.3772

\begin{abstract}
Protein tyrosine phosphatase receptor type $\mathrm{O}$ (PTPRO) has been recognized as a tumor suppressor in various types of cancer cells. However, little attention has been given to the role of PTPRO expression in the tumor microenvironment. We aimed to reveal the role of PTPRO in the breast cancer niche. Py8119 mouse breast cancer cells were implanted orthotopically into female wild-type or ptpro $^{-/}$C57Bl/6 mice. We observed that the loss of PTPRO in the tumor niche was correlated with larger tumor volume, more metastases, increased number of circulating tumor cells (CTCs), less apoptosis and reduced necrosis rates in the orthotopic mouse model of breast cancer. The tumor microenvironment in the ptpro $^{-/}$mice also showed increased microvessel density. Moreover, an intracardiac injection mouse model was used to determine the role of PTPRO in the pre-metastatic niche. Notably, more metastases were observed in the mice of the ptpro $^{-/-}$group. Taken together, PTPRO expression in the tumor niche prevents tumor growth and the formation of metastases of breast cancer, in part by attenuating tumor-associated angiogenesis and inducing the apoptosis and necrosis of tumor cells.
\end{abstract}

\section{Introduction}

Breast cancer, the most common malignancy and the second leading cause of cancer in women, is a heterogeneous disease

Correspondence to: Dr Shui Wang, Department of Breast Surgery, The First Affiliated Hospital of Nanjing Medical University, 300 Guangzhou Road, Nanjing, Jiangsu 210029, P.R. China

E-mail:ws0801@hotmail.com

*Contributed equally

Key words: protein tyrosine phosphatase receptor type O, breast cancer, tumor niche, tumor growth, metastasis with a variety of pathological entities (1). Approximately 232,340 new cases of invasive breast cancer were diagnosed in the USA in 2013, and almost 39,620 women will succumbed to this disease (2). The mechanism underlying tumor initiation and progression of this disease remain unclear, but partly due to deregulation of microenvironment homeostasis. The 'seed and soil' hypothesis indicates that an appropriate microenvironment (the soil) is crucial for the optimal growth of tumor cells (the seeds) (3).

Protein tyrosine phosphatase receptor type O (PTPRO), a type III member of the receptor-type PTP family, was initially reported to be expressed in human renal glomerulus (4). PTPRO is a transmembrane protein, and the PTP domain located in its intracellular region catalyzes the dephosphorylation of tyrosine peptides. Tyrosine phosphorylation plays a critical role in cellular processes such as cell proliferation, differentiation and apoptosis (5). PTPRO has attracted attention as an important tumor suppressor in multiple cancers. Overexpression of PTPRO was reported to induce the apoptosis of the terminally differentiated leukemic cell line U937 (6). It was also demonstrated that PTPRO acts as a tumor suppressor in human lung cancer cell line A549 (7). Furthermore, the promoter region of the PTPRO encoding gene is frequently methylated in human breast cancer as well as in other cancers, and this was found to be correlated with downregulation of PTPRO expression $(7,8)$.

However, to the best of our knowledge, most previous studies have focused on PTPRO expression in cancer cells while little attention has been given to the role of PTPRO expression in the tumor niche. In order to investigate the involvement of PTPRO in the breast cancer niche, we employed an orthotopic mouse mammary gland tumor model. As PTPRO was eliminated in the breast cancer niche, a significant increase in tumor size and the number of metastases were observed. These tumorsuppressive effects in the cancer niche were correlated with a reduction in tumor angiogenesis, circulating tumor cells (CTCs) and the induction of tumor apoptosis. Therefore, our results describe here for the first time, that PTPRO expression in the tumor niche represents a potential therapeutic strategy for breast cancer. 


\section{Materials and methods}

Cell culture. Py8119 cells were isolated from spontaneously arising tumors in MMTV-PyMT C57Bl/6 female mice by serial trypsinization and limiting dilution (9). Cells were cultured in F12K culture medium (Gibco-BRL, Grand Island, NY, USA) supplemented with $5 \%$ fetal bovine serum (FBS; Wisent, Nanjing, China) and MITO serum extender (BD Biosciences, Franklin Lakes, NJ, USA) and maintained in a humidified incubator at $37^{\circ} \mathrm{C}$ with $\mathrm{CO}_{2}$.

Animal experiments. The $\mathrm{ptpro}^{+/-} \mathrm{C} 57 \mathrm{Bl} / 6$ mice were kindly provided by Dr Bixby from the University of Miami. The PTPRO gene type was determined as described previously (10). Seven-week-old female C57Bl/6 mice and ptpro $^{-/-} \mathrm{C} 57 \mathrm{Bl} / 6$ mice (10 mice in each group) were used for the in vivo animal experiments. The animals were maintained under specific pathogen-free conditions in the animal housing facility of Nanjing Medical University. All animal protocols were approved and monitored by the Institutional Animal Care and Use Committee of Nanjing Medical University.

Orthotopic mammary tumor model. Py8119 cells tagged with luciferase-GFP (luciferase-GFP plasmid; kindly provided by Dr Brian Rabinovich, MD Anderson Cancer Center, Houston, TX, USA) were implanted orthotopically into the inguinal mammary fat-pad area $\left(10^{6}\right.$ cells/side/mouse $)$. The tumor size was measured with a caliper in 2 dimensions. Tumor volume was calculated with the equation: $\mathrm{V}=\left(\mathrm{L} \mathrm{x} \mathrm{W}^{2}\right) \times 0.5$, where $\mathrm{L}$ is the length and $\mathrm{W}$ is the width of the tumor. Six weeks after inoculation of the breast cancer cells, all mice were sacrificed and observed grossly by the Whole Body Imaging System (Illumatool 9900; Lightools Research, Encinitas, CA, USA). Lungs were removed during autopsy and GFP-expressing metastatic colonies were observed under a fluorescence microscope (Nikon, Melville, NY, USA).

Histology. Tumors and associated mammary glands were removed from the wild-type and $\mathrm{ptpro}^{+/} \mathrm{C} 57 \mathrm{Bl} / 6$ mice, fixed in $10 \%$ neutral buffered formalin and embedded in paraffin. Paraffin-embedded sections were stained with hematoxylin and eosin. For immunohistochemistry (IHC) staining, the specimens were deparaffinized, and rehydrated through xylene and graded alcohols. Sections mounted on slides were subjected to antigen retrieval by a pressure cooker, and then incubated with the antibodies specific for mouse CD31 and CD34 (both from Abcam, Cambridge, MA, USA). Immunocomplexes were visualized by the DAB method. All histologic analyses were examined by one pathologist. Image-Pro software (Media Cybernetics Inc., Rockville, MD, USA) was used to calculate the vessel density (vessels $/ \mathrm{mm}^{2}$ ). TUNEL staining was performed as described previously (11). Briefly, DNA fragmentation associated with apoptosis in the tumor cells was detected in situ by the addition of digoxigenin-labeled nucleotides to label the free 3'-end of DNA fragments using the Apoptosis Detection kit according to the manufacturer's instructions (Nanjing KeyGen Biotech Co., Ltd., Nanjing, China).

Circulating tumor cell detection. Six weeks after inoculation of the breast cancer cells in the mammary flat pads, blood was collected by cardiac puncture from the mice. All the procedures were performed under deep anesthesia with isofluorane. Eight hundred microliters of the blood sample was added to $25 \mathrm{ml} 1 \mathrm{X}$ red blood cell lysis buffer (BioLegend, San Diego, CA, USA). After gently vortexing, the samples were incubated at room temperature for $15 \mathrm{~min}$. Samples were washed with PBS, resuspended in $500 \mu 1$ PBS for flow cytometric analysis. Flow cytometric analysis was performed by using a BD FACSCalibur analyzer, and results were analyzed with CellQuest Pro software (both from BD Biosciences, San Jose, CA, USA).

Experimental in vivo metastasis study. An intracardiac injection model was used for the present study. Briefly, luciferase tagged py8119 cells were harvested and injected into the left cardiac ventricle of anesthetized female mice. Each mouse was injected with $2 \times 10^{5}$ cells in $0.1 \mathrm{ml}$ of phosphate-buffered saline. Development of metastasis induced by py 8119 cells was monitored at regular intervals by whole mouse fluorescence and bioluminescence imaging using the Xenogen IVISSpectrum System (Perkin-Elmer, Waltham, MA, USA).

Statistical analysis. All statistical analysis was carried out using GraphPad Prism 5 (GraphPad Software Inc., San Diego, CA, USA) and SPSS 16.0 software (IBM, Armonk, NY, USA). P-values were calculated by the Student's t-test and the Fisher's exact test. Data sets with $\mathrm{P} \leq 0.05$ were considered statistically significant.

\section{Results}

PTPRO expression in the tumor niche is essential for the inhibition of tumor growth and metastasis. We first determined the effect of PTPRO expression in the tumor niche on the regulation of tumorigenesis. GFP-expressing Py8119 mouse breast cancer cells were implanted orthotopically into female wild-type or ptpro $^{-/-} \mathrm{C} 57 \mathrm{Bl} / 6$ mice. After 2 weeks of inoculation, the incidence of tumor formation in both groups was $100 \%$. No significant differences in the frequency of tumor formation were observed.

We next investigated whether PTPRO expression in the niche could influence tumor growth and metastasis. Tumor size at the orthotopic site was measured by a vernier caliper at week 2, 4, and 6 after inoculation. The tumor growth rate in the 2 groups was initially similar, and the mean tumor volume of the 2 groups became significantly different after 4 weeks of inoculation (Fig. 1). Significantly larger tumors were observed in the $\mathrm{ptpro}^{-/-}$mice when compared with the wild-type mice, which indicate that PTPRO expression in the niche may act as a tumor suppressor. After the termination of the experiment, the numbers of peritoneum metastases (Fig. 2A) and bone metastases (Fig. 2B) were counted using the Whole Body Imaging System, and the metastatic tumors on the surface of the lung were observed under a fluorescence microscope (Fig. 2B). In the ptpro ${ }^{-/}$group, metastasis formed in most of the mice $(6 / 10)$, while in the wild-type group only one mouse was detected with metastasis (1/11) (Table I).

Increased vessel density in the ptpro-/ niche. Angiogenesis, regarded as a hallmark of cancer progression, is a multi-step 
A

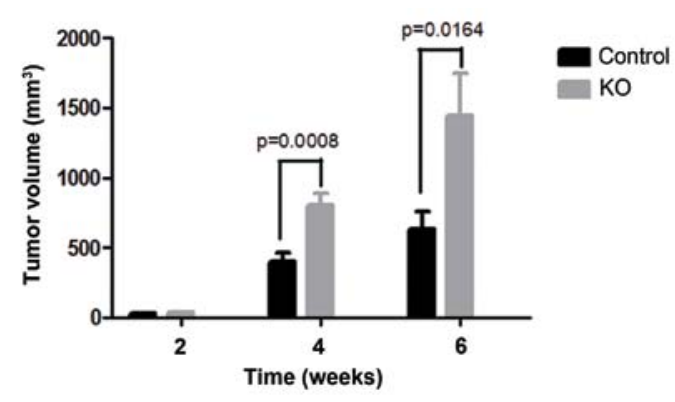

B

C

D
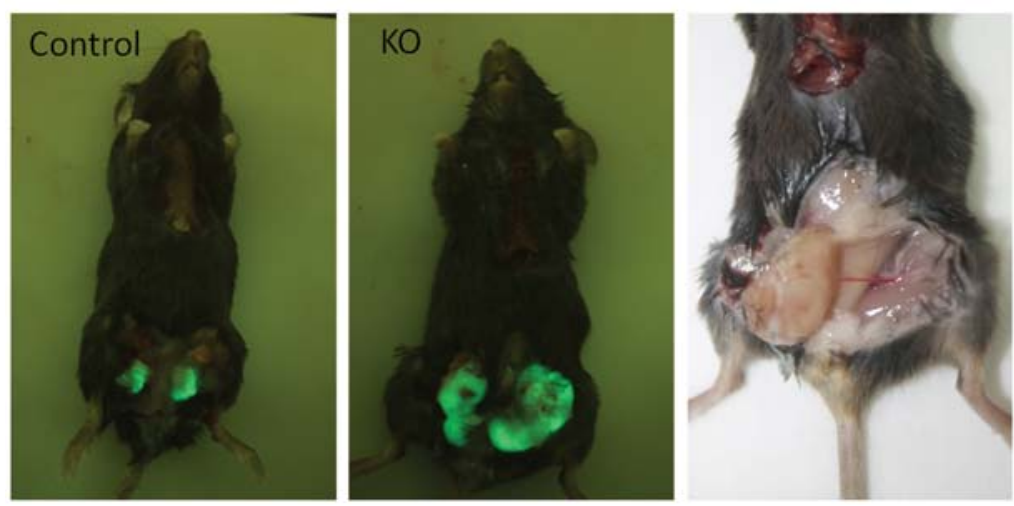

Figure 1. Increased tumor growth rates in the $\mathrm{ptpro}^{-/}$mice. (A) Tumor growth rates were significantly higher in the $\mathrm{ptpro}^{-/} \mathrm{mice}(\mathrm{KO})$ at week $4(\mathrm{p}=0.008)$ and week $6(\mathrm{p}=0.0164)$ when compared to the control. Representative whole body imaging of the tumor burden in the wild-type $(\mathrm{B})$ and $\mathrm{ptpro} \mathrm{O}^{-/-} \mathrm{C} 57 \mathrm{Bl} / 6(\mathrm{KO})(\mathrm{C})$ mice. (D) Enlarged tumor feeding branch blood vessel in a tpro $^{-/-}$C57Bl/6 mouse. PTPRO, protein tyrosine phosphatase receptor type O.
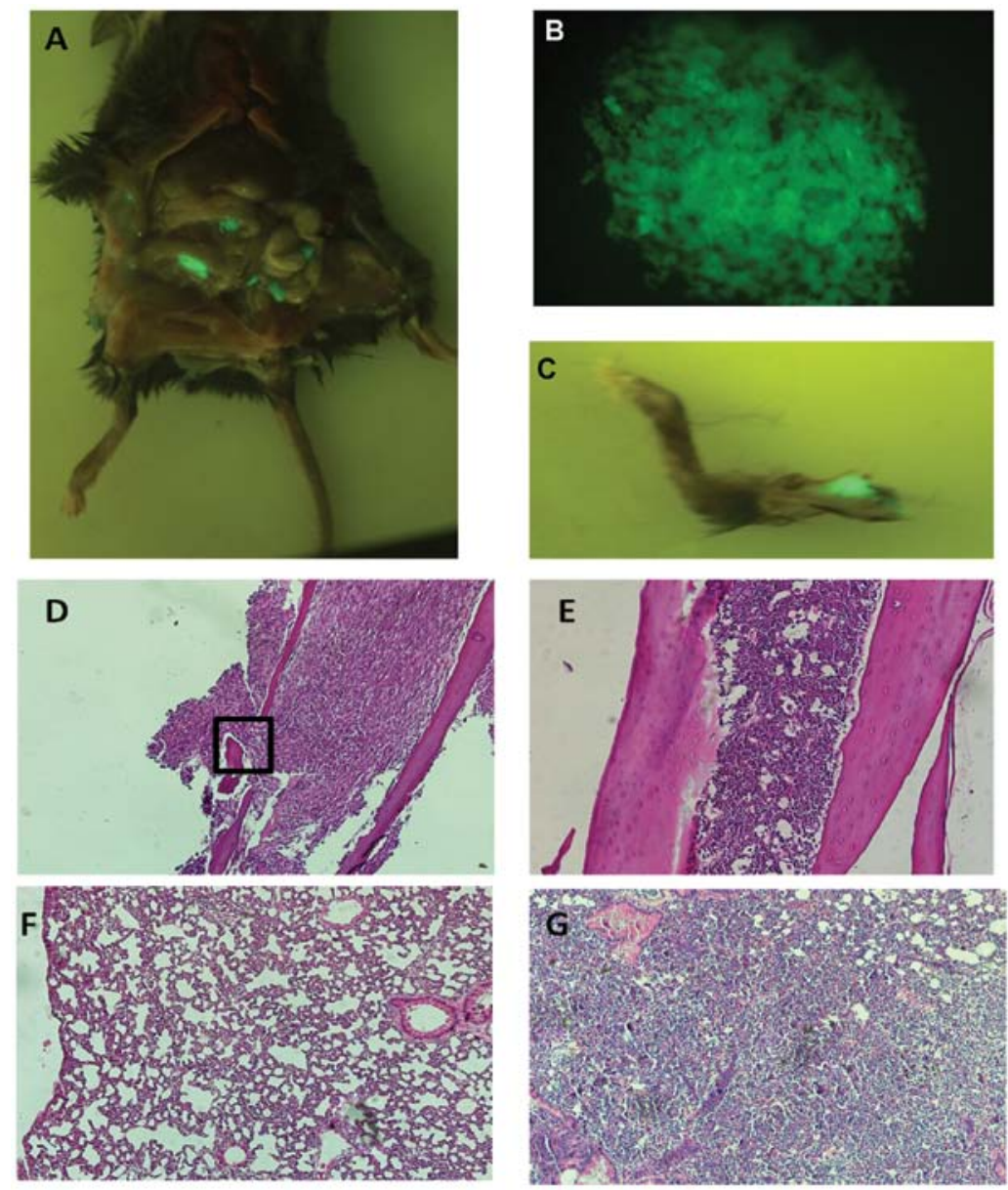

Figure 2. Increased metastasis in the ptpro ${ }^{-/}$mice in the orthotopic mouse model. Peritoneum metastases (A) and leg metastases (C) were observed grossly by the Whole Body Imaging System. (B) Metastatic nodules in the lung were observed under a fluorescence microscope. Representative H\&E-stained sections of normal leg (E), leg metastasis (D), normal lung (F) and lung metastasis (G). Original magnification, x100. PTPRO, protein tyrosine phosphatase receptor type O. 
Table I. Metastases observed in mice.

\begin{tabular}{lllll}
\hline & TM & PM & LM & BM \\
\hline WT & $1 / 11$ & $1 / 11$ & $0 / 11$ & $0 / 11$ \\
KO & $6 / 10^{\mathrm{a}}$ & $2 / 10$ & $3 / 10$ & $1 / 10$
\end{tabular}

${ }^{\mathrm{a}} \mathrm{p}=0.024 ; \mathrm{TM}$, total metastases; PM, peritoneum metastases; LM, lung metastases; BM, bone metastases.

process which consists of the development of new blood vessels from pre-existing ones and is essential for tumor growth and metastatic spread of tumor cells (12). Our results showed that PTPRO expression in the niche was essential for the inhibition of tumor growth and metastasis. Therefore, we aimed to determine the microvessel density in the tumors of wild-type and ptpro $^{-/}$mice. Immunohistochemical staining was performed with the CD31 (Fig. 3A and B) and CD34 (Fig. 3C and D) antibodies. Notably, the quantification of IHC data indicated that the microvessel density was significantly higher in the tumor tissues of the ptpro $^{-/}$group when compared with that in the wild-type group (Fig. 3E and F).

PTPRO expression in the tumor niche is necessary for induction of apoptosis and necrosis. To further investigate the role of PTPRO as a tumor inhibitor in the tumor niche, we also performed TUNEL assay to determine the apoptosis induced by PTPRO expression in the tumor niche. In wild-type mice, moderate numbers of apoptotic cells were clearly observed in the tumor tissues (Fig. 4C). In contrast, few apoptotic cells were noted in the tumor tissues of the ptpro $^{-/-}$mice (Fig. 4D). Importantly, the incidence of necrotic nodules was also higher in the tumor tissues of the wild-type mice (3/10) than that in the tissues ptpro $^{-/}$mice (0/10) (Fig. 4A and B).
A

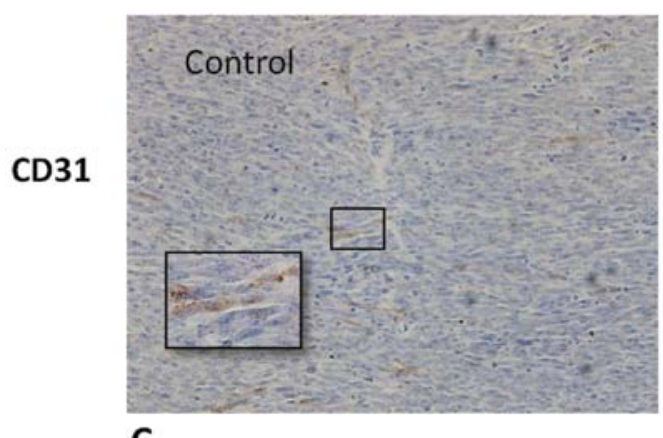

C

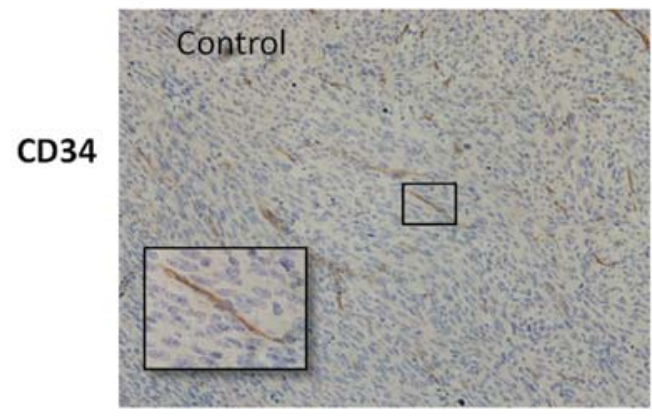

E

CD31 expression

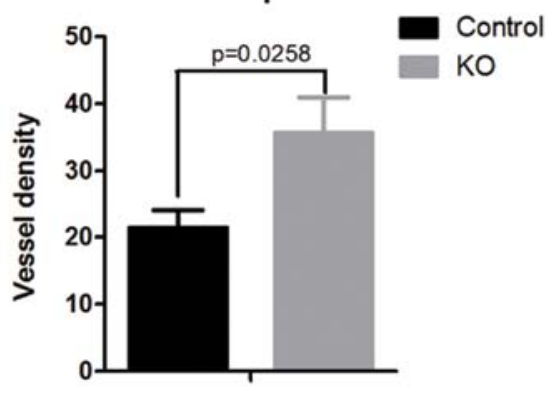

B

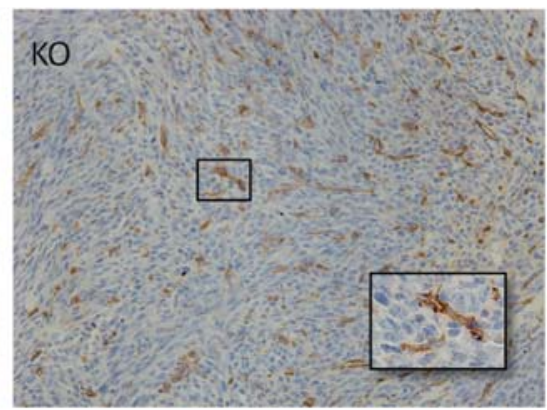

D

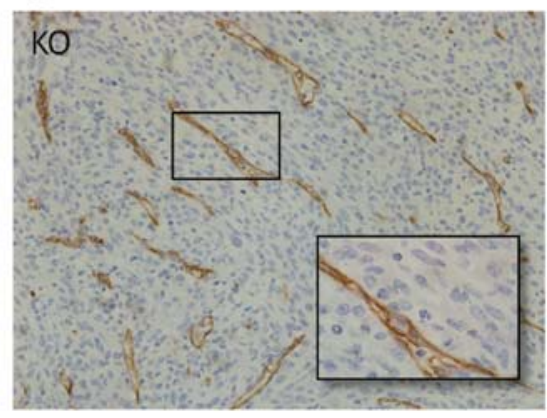

F

CD34 expression

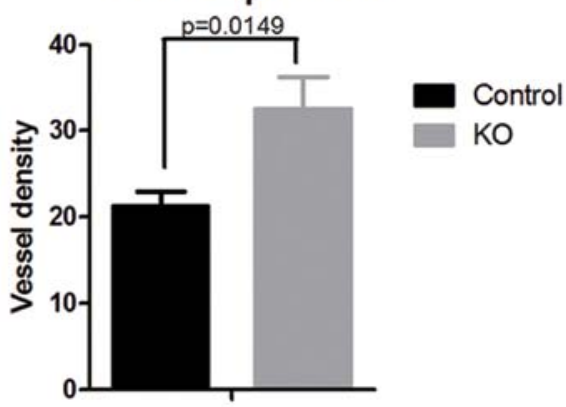

Figure 3. Increased microvessel density in tumors in the ptpro ${ }^{-/}$mice. Tumor tissues were removed from the tumor-burdened mice. Immunohistochemistry (IHC) staining for CD31 (A and B) and CD34 (C and D) was performed to evaluate the tumor-associated blood vessel density (vessels per mm ${ }^{2}$ ). (A) Significantly high level of CD31 (E, p=0.0258) and CD34 (F, p=0.0149) was observed in the tumor tissues of the ptpro ${ }^{-/}(\mathrm{KO}) \mathrm{mice}$. Original magnification, $\mathrm{x} 100 . \mathrm{PTPRO}$, protein tyrosine phosphatase receptor type $\mathrm{O}$. 
A

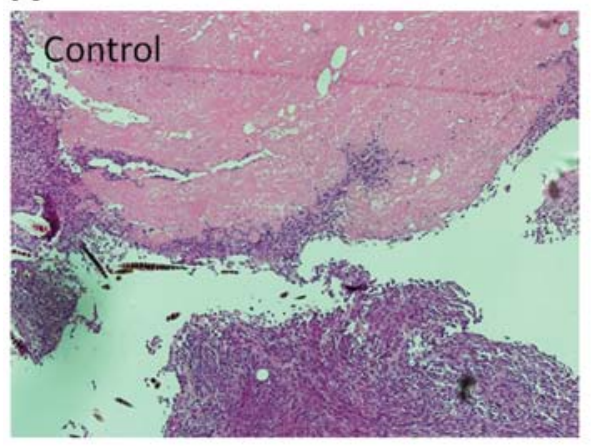

C

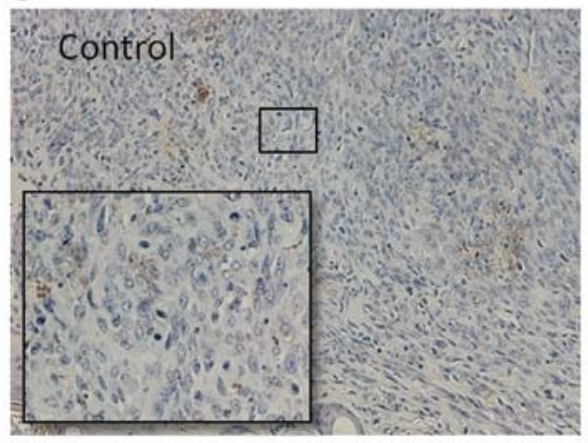

B

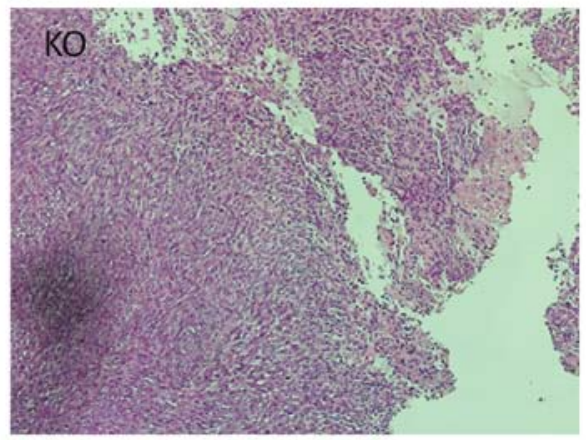

D

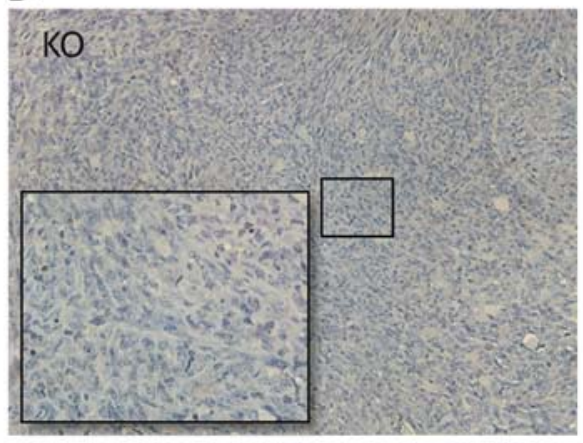

Figure 4. Less apoptotic and necrotic tissues were observed in the tumors of the ptpro ${ }^{-/}$mice. Representative H\&E-stained sections of necrotic tumor tissues in the wild-type mice (A) and that in the ptpro $^{-/}(\mathrm{KO})$ mice (B). Representative apoptotic cells as indicated in the tumor tissues of wild-type mice (C) and that in the ptpro $^{-/-}$mice (D). Original magnification, x100. PTPRO, protein tyrosine phosphatase receptor type O.

\section{Circulating tumor cells}

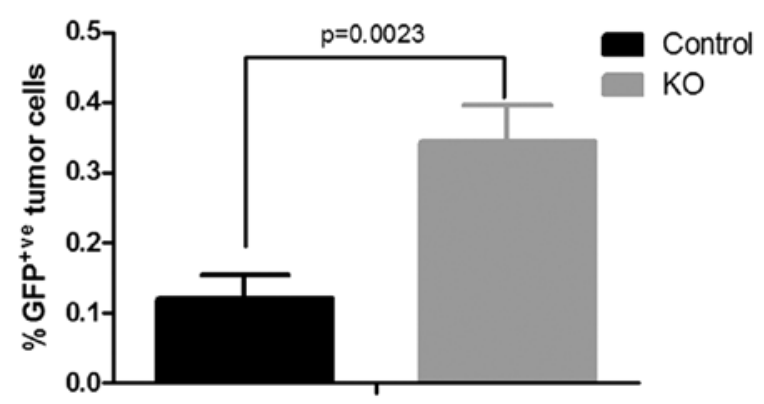

Figure 5. PTPRO deficiency in the tumor niche is correlated with increased CTC frequency: A significantly higher percentage of $\mathrm{GFP}^{+}$cells (CTCs) was observed in the blood of the ptpro ${ }^{-/}(\mathrm{KO})$ mice $(\mathrm{p}=0.0023)$. CTCs, circulating tumor cells; PTPRO, protein tyrosine phosphatase receptor type $\mathrm{O}$.

Escape of tumor cells from the primary tumor is facilitated by the ptpro $^{-/}$niche. Emerging evidence indicates that tumors are composed of tumor parenchyma and stroma, two discrete but interactive parts that cross-talk to regulate tumor growth and metastasis (13). Paracrine signaling between tumor cells and the tumor niche may play a critical role in the formation of metastasis. The fact that less metastasis was observed in the wild-type mice encouraged us to investigate the number of CTCs in the orthotopic tumor model. GFP-labeled Py8119 cells were inoculated into the mammary fat pad of the wild-type and ptpro $^{-/}$mice, and peripheral blood was then collected for calculation of CTCs by flow cytometry after 6 weeks. A significantly higher percentage of $\mathrm{GFP}^{+}$cells (CTCs) was observed in the blood of the ptpro $^{-/}$mice (Fig. 5). The results indicate that PTPRO expression in the tumor niche may prevent the escape of tumor cells from the primary tumor.

PTPRO expression in the premetastatic niche prevents the formation of metastasis. The 'seed and soil' hypothesis postulates that an appropriate host microenvironment is essential for the formation of metastasis (3). Therefore, we recruited the intracardiac injection model to investigate the direct effect of PTPRO expression in the pre-metastatic niche on metastasis formation. The luciferase-tagged Py8119 cells were inoculated intracardiacally into female wild-type or $\mathrm{ptpro}^{-/-} \mathrm{C} 57 \mathrm{Bl} / 6$ mice. The metastatic tumor growth was monitored at regular intervals by fluorescence and bioluminescence imaging. Notably, 4 weeks after the intracardiac injection, the signals of total flux in the ptpro $^{-/}$group were significantly higher than that in the control group (Fig. 6), which indicated that more metastases formed in the mice of the ptpro $^{-/-}$group. Our results showed that PTPRO expression may play an important role in the prevention of metastasis formation in the pre-metastatic niche.

\section{Discussion}

PTPRO, also known as glomerular epithelial protein 1 (GLEPP1), was initially identified in research for podocyte-specific proteins that may regulate glomerular structure and function (4). Subsequent studies indicated that PTPRO may act as a tumor suppressor in leukemia (8), hepatocellular carcinoma (14), lung cancer $(7)$ as well as breast cancer $(15,16)$. In a recent study, we also demonstrated that PTPRO in cancer 

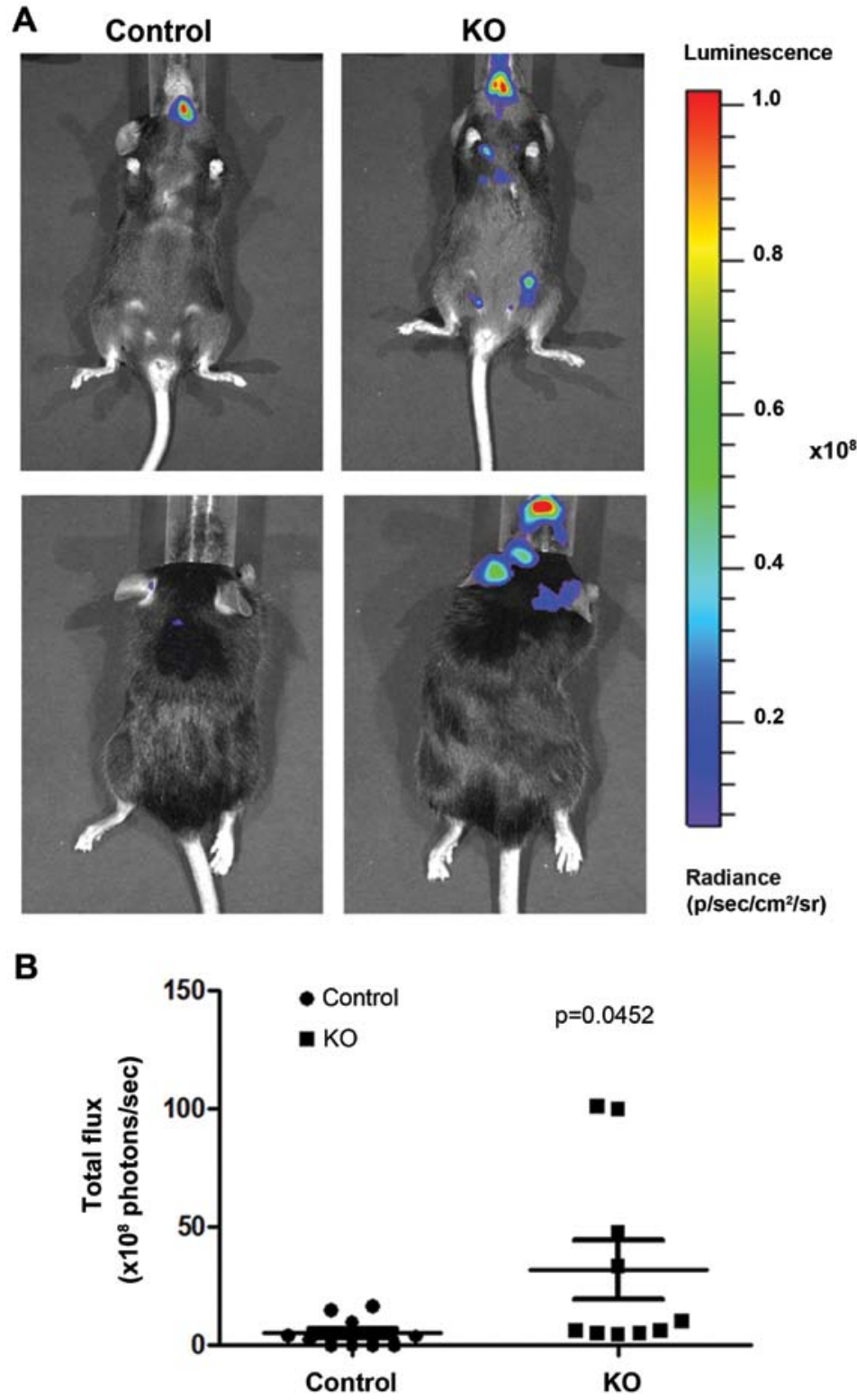

Figure 6. PTPRO expression in the pre-metastatic niche prevents the formation of metastasis. The luciferase-tagged Py8119 cells were inoculated intracardially into female wild-type or ptpro $^{-1-}$ (KO) C57Bl/6 mice. (A) The metastatic tumor growth was monitored by bioluminescence imaging. (B) Four weeks after the intracardiac injection, the signals of total flux in the ptpro $^{-/}$group $(\mathrm{KO})$ were significantly higher than that in the control group. PTPRO, protein tyrosine phosphatase receptor type $\mathrm{O}$.

cells could inhibit cell proliferation and enhance apoptosis by downregulation of signal transducers and activators of transcription 3 (STAT3) (10). In the past two decades, most investigators have focused primarily on the PTPRO expression in tumor cells. However, emerging evidence indicates that the tumor niche and microenvironment are important components which could cross-talk with tumors and modulate tumor growth (17,18).

Metastases are believed to be the major cause of death in breast cancer patients. It was reported that between 25 and $50 \%$ of patients diagnosed with breast cancer eventually develop deadly metastases (19). The formation of metastases requires the following key steps: tumor cell growth, angiogenesis, escape from the primary tumor, entrance into blood vessels, survival in vessels, and adaption to the premetastatic niche.
In the present study, we investigated the effect of PTPRO expression in the tumor niche on tumor growth, angiogenesis, CTCs and metastasis of breast cancer. Our results indicated that PTPRO expression in the tumor niche is essential for the inhibition of tumor growth. Significantly larger tumors and a higher metastasis frequency were observed in the $\mathrm{ptpro}^{-/}$mice when compared with the wild-type mice. We also found that the tumors harvested from the control mice showed more apoptosis and necrosis than that of the ptpro $^{-/}$group.

Angiogenesis promotes tumor growth and metastasis, and various PTPs have been implicated to regulate angiogenesis $(20,21)$. Thus, we investigated the vascularity in the tumors of the ptpro ${ }^{-/}$and control groups. CD31 (PECAM-1) and CD34 expression levels were recruited as markers for angiogenesis. Consistent with the tumor growth rate and the frequency of metastasis, we demonstrated that the microvessel density was 
significantly higher in the tumor tissues of the $\mathrm{ptpro}^{-/}$group when compared with that in the wild-type group. Therefore, we conclude that the increased tumor volume and frequency of metastasis in the ptpro $^{-/-}$group may be partly due to the loss of PTPRO signaling which could inhibit angiogenesis in the tumor microenvironment.

CTCs, rare malignant cells found in the peripheral blood, are useful as indicators of prognosis both initially and after therapy (22). Furthermore, the enumeration of CTCs could be a surrogate for the status of tumor cells in the circulating system. Our observation of increased numbers of CTCs in the ptpro $^{-/}$mice provides further evidence that PTPRO expression in the tumor niche inhibits tumor metastasis by preventing tumor cells from entering blood vessels. On the other hand, the formation of metastases is a highly inefficient process (23). From model systems, it has been estimated that $\sim 1 \times 10^{6}$ tumor cells per gram of tumor tissue can be introduced daily into the blood stream (24). The fate of CTCs is mainly determined by the acquirement of resistant to anoikis and the adaption to the new microenvironment of distant organs. To further explore the role of PTPRO expression in the pre-metastatic niche, we inoculated the same number of Py8119 cells intracardially into female wild-type or ptpro ${ }^{-/-} \mathrm{C} 57 \mathrm{Bl} / 6$ mice. Notably, more metastases were observed in the mice of the ptpro $^{-/}$group. These observations appear to indicate that PTPRO expression may play an important role in the prevention of metastasis formation in the pre-metastatic niche.

Our study had limitations. The tumor niche is composed of vascular endothelial cells, adipocytes, fibroblasts, platelets, mast cells and macrophages. We are not able to rule out the function of PTPRO in any particular cell type. Further studies are required to elucidate the role of PTPRO in different types of cells in the tumor niche.

In summary, our studies showed that PTPRO expression in the tumor niche acts as a tumor suppressor and inhibits the growth and metastasis of breast cancer cells. The mechanisms underlying the suppressive effect of PTPRO may be due to the induction of apoptosis and the suppression of angiogenesis.

\section{Acknowledgements}

We are grateful to Dr Lesley Ellies (University of California, San Diego) for providing us with the Py8119 cell line. The present study was supported in part by The National Natural Science Foundation of China (nos. 81270952, 81070684, $81071753,81172502,81202077$ and 81272916), The Natural Science Foundation of Jiangsu Province (BK2010581, BK2011853, BK2011855 and BK20141023), the Program for Development of Innovative Research Team in The First Affiliated Hospital of NJMU (IRT-008), a project funded by the Priority Academic Program Development of Jiangsu Higher Education Institutions (PAPD) and Jiangsu Province Innovation Project for Graduate Student (CXZZ13-0585).

\section{References}

1. Siegel R, Naishadham D and Jemal A: Cancer statistics, 2013. CA Cancer J Clin 63: 11-30, 2013.
2. Jemal A, Bray F, Center MM, Ferlay J, Ward E and Forman D: Global cancer statistics. CA Cancer J Clin 61: 69-90, 2011.

3. Paget S: The distribution of secondary growths in cancer of the breast. 1889. Cancer Metastasis Rev 8: 98-101, 1989.

4. Thomas PE, Wharram BL, Goyal M, Wiggins JE, Holzman LB and Wiggins RC: GLEPP1, a renal glomerular epithelial cell (podocyte) membrane protein-tyrosine phosphatase. Identification, molecular cloning, and characterization in rabbit. J Biol Chem 269: 19953-19962, 1994.

5. Jiang R, Xia Y, Li J, et al: High expression levels of IKKalpha and IKKbeta are necessary for the malignant properties of liver cancer. Int J Cancer 126: 1263-1274, 2010.

6. Seimiya $\mathrm{H}$ and Tsuruo T: Functional involvement of PTP-U2L in apoptosis subsequent to terminal differentiation of monoblastoid leukemia cells. J Biol Chem 273: 21187-21193, 1998.

7. Motiwala T, Kutay H, Ghoshal K, et al: Protein tyrosine phosphatase receptor-type O (PTPRO) exhibits characteristics of a candidate tumor suppressor in human lung cancer. Proc Natl Acad Sci USA 101: 13844-13849, 2004.

8. Motiwala T, Majumder S, Kutay H, et al: Methylation and silencing of protein tyrosine phosphatase receptor type $\mathrm{O}$ in chronic lymphocytic leukemia. Clin Cancer Res 13: 3174-3181, 2007.

9. Gibby K, You WK, Kadoya K, et al: Early vascular deficits are correlated with delayed mammary tumorigenesis in the MMTV-PyMT transgenic mouse following genetic ablation of the NG2 proteoglycan. Breast Cancer Res 14: R67, 2012.

10. Hou J, Xu J, Jiang R, et al: Estrogen-sensitive PTPRO expression represses hepatocellular carcinoma progression by control of STAT3. Hepatology 57: 678-688, 2013.

11. Liu Z, Bandyopadhyay A, Nichols RW, et al: Blockade of autocrine TGF- $\beta$ signaling inhibits stem cell phenotype, survival, and metastasis of murine breast cancer cells. J Stem Cell Res Ther 2: 1-8, 2012.

12. Carvalho MI, Guimaraes MJ, Pires I, et al: EGFR and microvessel density in canine malignant mammary tumours. Res Vet Sci 95: 1094-1099, 2013.

13. Mao Y, Keller ET, Garfield DH, Shen K and Wang J: Stromal cells in tumor microenvironment and breast cancer. Cancer Metastasis Rev 32: 303-315, 2013.

14. Motiwala T, Ghoshal K, Das A, et al: Suppression of the protein tyrosine phosphatase receptor type O gene (PTPRO) by methylation in hepatocellular carcinomas. Oncogene 22: 6319-6331, 2003.

15. Huang YT, Li FF, Ke C, et al: PTPRO promoter methylation is predictive of poorer outcome for HER2-positive breast cancer: indication for personalized therapy. J Transl Med 11: 245, 2013.

16. Li SY, Li R, Chen YL, et al: Aberrant PTPRO methylation in tumor tissues as a potential biomarker that predicts clinical outcomes in breast cancer patients. BMC Genet 15: 67, 2014.

17. Derycke L, Van MV, Depypere H and Bracke M: Molecular targets of growth, differentiation, tissue integrity, and ectopic cell death in cancer cells. Cancer Biother Radiopharm 20: 579-588, 2005.

18. Kopfstein L and Christofori G: Metastasis: cell-autonomous mechanisms versus contributions by the tumor microenvironment. Cell Mol Life Sci 63: 449-468, 2006.

19. Lorusso $G$ and Ruegg C: New insights into the mechanisms of organ-specific breast cancer metastasis. Semin Cancer Biol 22: 226-233, 2012.

20. Cai J, Chen Z, Ruan Q, et al: $\gamma$-secretase and presenilin mediate cleavage and phosphorylation of vascular endothelial growth factor receptor-1. J Biol Chem 286: 42514-42523, 2011.

21. Chen JX, Tuo Q, Liao DF and Zeng H: Inhibition of protein tyrosine phosphatase improves angiogenesis via enhancing Ang-1/Tie-2 signaling in diabetes. Exp Diabetes Res 2012: 836759, 2012.

22. Alemar J and Schuur ER: Progress in using circulating tumor cell information to improve metastatic breast cancer therapy. J Oncol 2013: 702732, 2013.

23. Paterlini-Brechot P and Benali NL: Circulating tumor cell (CTC) detection: clinical impact and future directions. Cancer Lett 253: 180-204, 2007.

24. Chang YS, di Tomaso E, McDonald DM, Jones R, Jain RK and Munn LL: Mosaic blood vessels in tumors: frequency of cancer cells in contact with flowing blood. Proc Natl Acad Sci USA 97: 14608-14613, 2000. 